\title{
EDITORIAL
}

\section{Central pain contributions in osteoarthritis: next steps for improving recognition and treatment?}

Kelli Allen*

See related research by Murphy et al., http://arthritis-research.com/content/13/4/R135

\begin{abstract}
There is increasing recognition that central pain sensitivity plays an important role in pain severity among patients with osteoarthritis. Murphy and colleagues identified clusters of patients with osteoarthritis according to pain severity and accompanying symptoms, and one of these groups appeared to have a greater degree of centrally mediated pain. This observation provides some evidence that patients with greater central pain contributions can be identified in routine clinical settings, but brief, evidence-based strategies are still needed to more readily and systematically identify these patients. There is also a need to develop new strategies and to further evaluate existing therapies (pharmacological and nonpharmacological) that target central aspects of osteoarthritis pain.
\end{abstract}

Much is still not understood regarding mechanisms of osteoarthritis (OA)-related pain. Studies have found differing degrees of association between radiographic changes and pain severity, but it is generally accepted that factors other than radiographic features contribute to pain levels. Damage or inflammation of joint structures such as the synovial capusule, bursae, fat pad, ligaments, menisci, or adjacent bone may contribute to pain severity.

There is increasing recognition of the important role of central pain sensitivity, however, which is the focus of the recent paper by Murphy and colleagues in Arthritis Research and Therapy [1]. The hypothesis is, and there are data to support, that chronic OA-related peripheral pain stimuli may lead to hyperexcitability of paintransmitting neurons [2]. Studies have shown that

*Correspondence: kelli.allen@duke.edu

Department of Medicine, Duke University Medical Center, Health Services Research \& Development, Durham VA Medical Center, 508 Fulton Street, Durham, NC 27705, USA patients with $\mathrm{OA}$ do experience heightened pain sensitivity, with substantial intra-individual variability $[2,3]$. However, the vast majority of OA-related treatments target the periphery; for example, nonsteroidal anti-inflammatory drugs act on local inflammatory processes. While these medications also affect central pain mechanisms - either directly or indirectly through their influence on peripheral pain stimuli and sensitivity [4] - failure to more fully address central pain sensitization may be one reason for inadequate pain relief in some patients.

The study by Murphy and colleagues provides additional support that patients differ in the degree to which central mechanisms contribute to OA-related pain and other symptoms [1]. The authors used a cluster analysis approach and identified three subgroups of patients, one of which was characterized not only by the highest pain levels but also by other symptoms characteristic of centrally mediated pain. This is an interesting and clinically relevant approach to the question of central pain contribution, providing support to the notion that patients with greater central pain sensitivity could be identified in routine care settings, without the use of formal pain threshold testing or other processes that would typically be utilized in research.

Given the mounting evidence for the importance of central pain sensitivity in OA [5], how can this be addressed in routine clinical care? There is certainly a need to further educate clinicians (and particularly those in primary care, who typically provide first-line treatment for OA) about the important role of central pain adaptations, basic signs of central pain sensitivity, and the utility of current treatments to address this aspect of pain. Cognitive behavioral pain management interventions have shown efficacy for treating neuropathic pain, but these interventions are underutilized among patients with OA. Increased availability and use of these programs could be one practical step toward improving treatment of the central aspect of pain. Opioid analgesics act centrally and also have a role in OA treatment; despite their potential for adverse side effects, they may be an effective treatment for patients with a strong central 
contribution to the pain experience. There is also some evidence for use of duloxetine and other antidepressants in the treatment of OA-related pain, and these therapies may be most effective for patients with greater central contributions to pain. More data are needed, however, to determine the appropriateness and effectiveness of these medications in the treatment of $\mathrm{OA}$, as well as guidelines for when and how they are best applied in this patient group.

Murphy and colleagues and other researchers have suggested that OA treatment regimens could be tailored according to patients' pain mechanisms (for example, relative contribution of peripheral vs. central contributions). This suggestion certainly seems appealing both in terms of clinical efficiency and likelihood of treatment response. However, there are at least two areas in which additional research is needed to support this tailored treatment becoming a clinical reality. First, additional research is needed to both identify new strategies and further evaluate existing therapies (including pharmacological and nonpharmacological) that target central aspects of OA-related pain. This area of treatment development has lagged considerably behind strategies to address peripheral aspects of pain. Second, brief and evidence-based strategies are needed to identify patients who have a stronger central component to OA-related pain. The study by Murphy and colleagues suggests this further research is possible, and other studies - for example, that by Gwilym and colleagues [6] - have used surveys to identify patients with neuropathic pain symptoms. The existing screening tools are probably too cumbersome for busy care providers, however, and simpler methods are needed for clinicians to readily identify patients who may benefit more from treatments that target central pain mechanisms.

In summary, treatment of central pain sensitivity is an area with great promise for improving pain management in at least a subset of patients with OA; efforts are clearly needed to expand the evidence base for both identification and treatment of central pain contributions.

\section{Abbreviations}

OA, osteoarthritis.

\section{Competing interests}

The author declares that she has no competing interests.

Published: 25 November 2011

\section{References}

1. Murphy SL, Lyden AK, Phillips K, Clauw DJ, Williams DA: Subgroups of older adults with osteoarthritis based upon differing comorbid symptom presentations and potential underlying pain mechanisms. Arthritis Res Ther 2011, 13:R135.

2. Kosek E, Ordeberg G: Abnormalities of somatosensory perception in patients with painful osteoarthritis normalize following successful treatment. Eur J Pain 2000, 4:229-238.

3. Hochman JR, French MR, Bermingham SL, Hawker GA: The nerve of osteoarthritis pain. Arthritis Care Res 2010, 62:1019-1023.

4. Burian M, Geisslinger G: COX-dependent mechanisms involved in the antinociceptive action of NSAIDs at central and peripheral sites. Pharmacol Ther 2005, 107:139-154.

5. Mease PJ, Hanna S, Frakes EP, Altman RD: Pain mechanisms in osteoarthritis: understanding the role of central pain and current approaches to its treatment. J Rheumato/ 2011, 38:1546-1551.

6. Gwilym SE, Keltner JR, Warnaby CE, Carr AJ, Chizh B, Chessell I, Tracey I: Psychophysiological and functional imaging evidence supporting the presence of central sensitization in a cohort of osteoarthritis patients. Arthritis Care Res 2009, 61:1226-1234.

doi:10.1186/ar3499

Cite this article as: Allen K: Central pain contributions in osteoarthritis: next steps for improving recognition and treatment? Arthritis Research \& Therapy 2011, 13:133. 